\title{
Dynamical Simulations of the Shaping of PNe including Radiation Effects
}

\section{J. Zweigle}

Institut de Radio Astronomie Millimétrique (IRAM)

In order to investigate the shaping of aspherical planetary nebulae the gasdynamical conservation laws of mass, momentum and energy including radiation effects are solved numerically in twodimensional, spherical coordinates. The simulations describe the dynamical interaction of a fast, tenuous, spherically symmetric wind from the central star with the slow, dense, axisymmetric AGB-wind remnant and include photoionization, collisional ionization, collisional excitation, radiative recombination and forbidden line emission as radiation effects.

For comparison with observations monochromatic images and high resolution long slit spectra in the $\mathrm{H} \alpha$-emission line are calculated for different inclination angles. The model images and spectra show striking similarities to corresponding observations of planetary nebulae.

Under the assumption, that the hot bubble, originating from the dynamical interaction of the two winds, emits optically thin thermal bremsstrahlung, its emission is modelled in the energy range from 0.1 to $2.4 \mathrm{keV}$. For a terminal central star wind velocity of $2000 \mathrm{~km} / \mathrm{s}$ the calculated $\mathrm{x}$-ray spectrum of the isobaric, anisothermal bubble can be approximated very well by an isothermal bremsstrahlung spectrum with a temperature of $5 \cdot 10^{6} \mathrm{~K}$. This value is approximately ten times smaller than the maximum temperature in the hot bubble and in the same order as the plasma temperatures derived from ROSAT observations of PNe, e.g. $2.3 \cdot 10^{6} \mathrm{~K}$ for $\mathrm{BD}+30^{\circ} 3639$ and $1.5 \cdot 10^{6} \mathrm{~K}$ for NGC 6543 (Kreysing et al. 1992).

\section{REFERENCES}

Kreysing H.C. et al., 1992, Astron. Astrophys., 264, 623. 